\title{
Consumption vs. deposition of essential fatty acids in gilthead sea bream (Sparus aurata) larvae fed semi-purified diets
}

\author{
Jean H. Robin * and Alexandre Peron
}

Unité mixte Nutrition, Aquaculture, Génomique INRA-IFREMER-Bordeaux I, Centre IFREMER de Brest, BP 70, 29280 Plouzané, France

*: Corresponding author : jrobin@ifremer.fr

\begin{abstract}
Semi-purified diets were used to feed gilthead sea bream larvae as first food from mouth opening until day 17. Four diets were formulated on the basis of a previous one used as control (D1). Three experimental diets differed only by the lipids added. One diet contained n-3 highly unsaturated fatty acids ( $\mathrm{n}-3$ HUFA) plus arachidonic acid (D2), one diet n-3 HUFA without arachidonic acid (D3) and one diet without n-3 and n-6 HUFA (D4). The three experimental diets contained 18:3n-6 in order to follow the influence of dietary HUFAs on elongation-desaturation capacities. Larval growth and survival were similar with diet D1 and D2. Lowest growth and survival were observed for larvae fed the HUFA free diet D4. Larval survival but not growth was significantly decreased by lack of arachidonic acid in diet D3 compared to D2. Utilization of fatty acids by larvae was studied by comparing the fatty acid quantities per larva in initial (before feeding) and final samples. In larvae fed the HUFA-free diet D4, a significant increase of 20:3n-6 showed that some elongation from 18:3n-6 occurred, while quantities of arachidonic acid and n-3 fatty acids (except 18:3n-3) decreased compared to initial larvae. A consumption of endogenous HUFAs was evidenced despite effective incorporation of dietary fatty acids such as $18: 2 n-6,18: 1 n-9$ and $18: 3 n-3$. Fatty acid profile of these larvae changed, according to dietary fatty acid influence, by turnover rather than by a raw incorporation of fatty acids. Larvae fed the n-3 HUFA diet depleted in arachidonic acid (D3) had a total fatty acid content lower than those fed with arachidonic acid (D2); compared to those fed D4, the elongation of $18: 3 n-6$ to $20: 3 n-6$ seemed to be repressed by dietary n-3 HUFA.

Consumption of long-chain unsaturated fatty acid is a main conclusion of this study; a rate of loss around 1.1, 0.5 and $0.05 \mathrm{mg}$ day $-1 \mathrm{~g}-1$ mean dry body weight of larvae for docosahexaenoic, eicosapentaenoic and arachidonic acid, respectively, may be estimated in larvae fed the HUFA free diet D4. No desaturation capacities of gilthead sea bream larvae were evidenced; however, some low capacities might be masked by the consumption of HUFAs.
\end{abstract}

Keywords: Sparus aurata; Larva; Inert diet; Fatty acids 


\section{Introduction}

For gilthead sea bream larva, inert diets have been successfully used after a few days of feeding live prey (Yúfera et al., 2000) or in co-feeding with live prey (Koven et al., 2001). Significant growth of gilthead sea bream larva has also been obtained by using compound or semi-purified diets as first food (Robin and Vincent, 2003). Beside the development of practical diets useful for juvenile production (Cahu and Zambonino-Infante, 2001), the use of purified diets is extremely useful as a tool to study the nutritional requirements in larval stages. Such an approach was successfully used in the nutrition of larvae of some fresh water species (Radünz-Neto et al., 1994, 1996) using casein-based micro-particulate diets.

Like most marine fish, gilthead sea bream was considered to have an absolute requirement for highly unsaturated fatty acids (HUFA) : eicosapentaenoic acid EPA (20:5n-3), docosahexaenoic acid DHA (22:6n-3) and arachidonic acid ARA (20:4n-6), being unable to produce these fatty acids from the precursors 18:3n-3 and 18:2n-6 (Sargent et al., 1995). Such transformation of fatty acids imply the activity of specific enzymes : $\Delta 6$ - and $\Delta 5$-desaturases (de Antueno et al., 2001) and elongases (Leonard et al., 2004). As typical marine fish always find preformed HUFA in their natural preys, it was assumed that they have lost bioconversion capacities, while salmonids born in freshwater have maintained efficient fatty acid transformation capacities (Kanazawa, 1985). In turbot, Ghioni et al. (1999) observed a limiting elongation capacity of $\mathrm{C} 18$ to $\mathrm{C} 20$ polyunsaturated fatty acids, but desaturation occurred. In fasted gilthead sea bream, desaturation capacities were evidenced by Mourente and Tocher (1994). Recently, Seiliez et al. (2003) identified a $\Delta 6$-desaturase like gene in this species, in fish fed a HUFA-free diet, but not in fish fed a HUFA-rich diet. Even in salmonids a diet providing HUFA seems to repress desaturase expression (Ruyter and Thomassen, 1999). 
The present study was designed in order to observe if polyunsaturated fatty acid transformation could be enhanced during first feeding by a HUFA free diet. Semi-purified diets were formulated on the basis of a previous one (Robin and Vincent, 2003) used as control (D1), and three others modified in order to have a common basis and different lipid supplement. These experimental diets differed by the lipid sources added in order to compare a diet containing n-3 HUFA plus ARA (D2), a diet containing n-3 HUFA without ARA (D3) and a HUFA free diet (D4).

\section{Material and methods}

\subsection{Experimental fish and diets:}

A batch of newly hatched larvae of gilthead sea bream (Sparus aurata) was obtained from "Ferme marine du Douhet" (La Brée les Bains, France). Larvae were randomly distributed in 351 cylindro-conical tanks (5800 larvae per tank by volumetric counting, controlled by individual counting of 3 extra tanks $)$. A flow $\left(0.71 . \mathrm{min}^{-1}\right)$ of UV-treated, filtered $(5 \mu \mathrm{m})$ seawater (34-35\%) was applied to each tank with temperature maintained at $20^{\circ} \mathrm{C}$.

Larvae were kept under darkness until mouth opening at day 3 post hatch, then light was progressively increased up to 450 lux. Then diets, with three replicates per treatment, were distributed at $2 \mathrm{~g} \mathrm{tank}^{-1} \mathrm{day}^{-1}$ continuously with automatic feeders. Excess food, deposited on tank bottom, was removed by daily siphoning. At the end of the experiment (day 17) larvae were kept unfed overnight. Samples of 30 larvae were preserved in 5\% formalin and weighed, then the larvae were dried in an oven at $110^{\circ} \mathrm{C}$ during $24 \mathrm{~h}$ for dry weight measurement. Remaining larvae were counted and samples of a known quantity $(300)$ were stored at $-80^{\circ} \mathrm{C}$ until analysis. Three samples of larvae on day 3 (before first feeding) and 2 samples of larvae starved until day 8 were also analysed by the same way. 
Four diets were prepared using ingredients presented in Table 1, and pelletized according to Fontagné et al. (2000). Ingredients were finely ground below $100 \mu \mathrm{m}$, and mixed before addition of oil and water. The moist blend was pelletized ( $3 \mathrm{~mm}$ diameter) using a meat grinder. Pellets were dried on air flux at $50^{\circ} \mathrm{C}$ during $20 \mathrm{~min}$, ground and sieved in order to obtain 50-160 $\mu \mathrm{m}$ particles. Diet 1 , used as control, was quite similar to the semi-purified diet used in Robin and Vincent (2003) but egg lecithin was used instead of soy lecithin. The other diets contained Spirulina instead of algamac and the quantities of other ingredients were calculated to obtain similar protein and lipid contents. Casein, casein hydrolysate and caseinate sodium salt were used as main protein source according to Carvalho et al. (1997).

A high phospholipid quantity (Cahu et al., 2003) was provided either by egg lecithin (D1 and D2) or by soybean lecithin (D3 and D4). Cod liver oil was used in D1. A HUFA concentrate associated with olive oil was used for D2 and D3 in order to obtain DHA/EPA ratios superior to 2 (Rodriguez et al., 1998). Ingredients used for D 4 were free of n-3 and n-6 HUFAs. 
Table 1 : Components $\left(\mathrm{g} \cdot \mathrm{kg}^{-1}\right)$ of experimental diets.

\begin{tabular}{lcccc}
\hline diets & D1 & D2 & D3 & D4 \\
\hline Algamac & 140 & - & - & - \\
Spirulina & - & 140 & 140 & 140 \\
Dextrins & 60.0 & 88.0 & 88.0 & 88.0 \\
Casein hydrolysate & 42.0 & 36.6 & 36.6 & 36.6 \\
Caseinate sodium salt & 105 & 91.7 & 91.7 & 91.7 \\
Casein & 357 & 311.7 & 311.7 & 311.7 \\
Ascorbyl palmitate & 3 & 3 & 3 & 3 \\
Vitamin mix & 77 & 77 & 77 & 77 \\
Amino acids & 41 & 36 & 36 & 36 \\
Mineral mix & 40 & 40 & 40 & 40 \\
Betain & 10 & 10 & 10 & 10 \\
Ovothin 160 & 100 & 100 & - & - \\
Soybean lecithin & - & - & 100 & 100 \\
Cod liver oil & 25 & - & - & - \\
EPAX (20-50) & - & 25.0 & 28.5 & - \\
Olive oil & - & 41.0 & 37.5 & 66.0 \\
\hline
\end{tabular}

Sources: Algamac : Aquafauna Biomarine (Hawthorne, Ca, USA); casein C7078, casein sodium salt C8654, casein hydrolysate NZ amine: C0626 Sigma; Ovothin 160 : Degussa ; Soybean lecithin LPR: SAPA DADA S.D.A (Marne la Vallée, France); olive oil: Amphora (Chateau Gontier, France), EPAX 20-50 Pronova Biocare; Vitamin and mineral mixture as in Fontagné et al. (2000).

\subsection{Analysis}

Proximate analyses of diets were made following standard methods : dry matter after desiccation in an oven $\left(105^{\circ} \mathrm{C}\right.$ for $\left.24 \mathrm{~h}\right)$, ash (incineration at $550^{\circ} \mathrm{C}$ for $\left.12 \mathrm{~h}\right)$, crude protein (Dumas, Nitrogen Analyser, Fison Instrument, $\mathrm{N} \times 6.25$ ), lipids were extracted and quantified according to Folch et al. (1957). For lipid analyses of larvae, an internal standard (tricosanoic acid 23:0) was added on a sample of known quantity of larvae, then extraction was done according to Folch et al. (1957). A part of each lipid sample (1 mg) was transmethylated according to Morrison and Smith (1964) for total fatty content, with $2 \mathrm{ml}$ of $(1: 1: 1)$ methanol:toluene: $10 \% \mathrm{BF}_{3}$-methanol. The other part was used to separate polar lipid from neutral lipids according to Juaneda and Roquelin (1985) on sep-pack light cartridges (Waters 
023537), and then also transmethylated. All glassware used for extraction and transmethylation were heated at $550^{\circ} \mathrm{C}$ in an oven over night, before use. Fatty acid methyl esters were separated by gas chromatography as described previously in Regost et al. (2002). The FA contents per larva were quantified by reporting the area of peaks for each fatty acid to the area of the internal standard and dividing by the number of larvae analysed. A reference sample of cod liver oil transmethylated with the internal standard was regularly injected to control the regularity of the quantitative responses.

\subsection{Statistical analysis:}

Comparisons of effects of dietary treatments were performed by one way ANOVA.

Significant differences $(\mathrm{P}<0.05)$ between means were compared by the Newman-Keuls test. Data on survival and fatty acid percentages were transformed by arcsinus of square root before applying ANOVA. 


\section{Results}

The fatty acid composition of the diets (Table 2) corresponded to the expected experimental design. Diets 1 to 3 contained n-3 HUFA near 1.7\% DM. D 3 was almost free of arachidonic acid $(0.02 \% \mathrm{DM})$ whereas egg lecithin induced substantial arachidonic contents $(0.26-$ $0.25 \%$ DM) in D1 and D2, while D4 was free of n-3 and n-6 HUFAs. Diets were calculated in order to be isoproteic and isolipidic, however, as shown in Table 2, that was not fully obtained.

Table 2 Proximal analysis and fatty acid content of diets used.

\begin{tabular}{|c|c|c|c|c|}
\hline diets & D1 & $\mathrm{D} 2$ & D3 & D4 \\
\hline Protein \%DM & 57.8 & 57.7 & 58.3 & 58.4 \\
\hline Lipids \%DM & 18.1 & 16.9 & 14.1 & 14.7 \\
\hline \multicolumn{5}{|l|}{ FA ( $\%$ area) } \\
\hline $14: 0$ & 8.5 & 0.3 & 0.3 & 0.2 \\
\hline $16: 0$ & 31.2 & 19.7 & 14.3 & 16.7 \\
\hline 18:0 & 6.4 & 6.5 & 3.0 & 3.7 \\
\hline 20:0 & 0.1 & 0.2 & 0.2 & 0.3 \\
\hline $22: 0$ & 0.1 & 0.0 & 0.3 & 0.3 \\
\hline $16: 1 n-7$ & 3.7 & 1.3 & 0.6 & 0.7 \\
\hline $18: 1 n-7$ & 2.3 & 1.4 & 1.3 & 1.5 \\
\hline 18:1n-9 & 15.6 & 35.0 & 26.1 & 41.7 \\
\hline 20:1n-9 & 1.4 & 0.4 & 0.4 & 0.2 \\
\hline $22: 1 n-11$ & 1.0 & 0.0 & 0.1 & 0.0 \\
\hline $18: 2 n-6$ & 8.1 & 11.8 & 27.6 & 29.1 \\
\hline $18: 3 n-6$ & 0.1 & 1.4 & 1.4 & 1.4 \\
\hline $20: 2 n-6$ & 0.2 & 0.2 & 0.2 & 0.1 \\
\hline $20: 3 n-6$ & 0.2 & 0.2 & 0.1 & 0.0 \\
\hline $20: 4 n-6$ & 2.1 & 2.4 & 0.3 & 0.0 \\
\hline $22: 5 n-6$ & 3.3 & 0.5 & 0.4 & 0.2 \\
\hline $18: 3 n-3$ & 0.3 & 0.7 & 4.1 & 4.1 \\
\hline $18: 4 n-3$ & 0.3 & 0.4 & 0.4 & 0.0 \\
\hline $20: 3 n-3$ & 0.0 & 0.1 & 0.1 & 0.0 \\
\hline $20: 4 n-3$ & 0.3 & 0.3 & 0.4 & 0.0 \\
\hline $20: 5 n-3$ & 1.6 & 4.8 & 5.7 & 0.0 \\
\hline $22: 5 n-3$ & 0.3 & 1.0 & 1.1 & 0.0 \\
\hline $22: 6 n-3$ & 12.1 & 11.3 & 11.8 & 0.0 \\
\hline sum HUFA ( $\%$ FA) & 14.0 & 17.1 & 18.6 & 0 \\
\hline DHA/EPA & 7.61 & 2.35 & 2.07 & \\
\hline n-3HUFA\%DM & 1.7 & 1.8 & 1.6 & 0 \\
\hline $20: 4 n-6 \% D M$ & 0.26 & 0.25 & 0.02 & 0 \\
\hline
\end{tabular}


Observation of gut contents of larvae showed a good acceptance of all the particulate diets. There was a sudden onset of mortality in one tank of treatment D1 and one tank of treatment D2 before the end of the experiment, related to high development of filamentous bacteria in these tanks, inducing oxygen depletion and overflow by clogging outlet filters. This incidence on final survival was considered as accidental as the larvae were apparently healthy and at high density before this event. If these tanks are removed a statistical difference in percent survival can be observed (Table 3), a lower survival with treatment 4 can be evidenced, with a higher survival with D1 and D2 compared to D3 and D4. Diet 4 resulted in a lower final wet weight than other diets, and a lower dry weight, but in this case not significantly differing from D3.

Table 3: Mean body weight before feeding (day3) and at day 17 of larvae fed the various diets. Mean and standard error $(n=3)$.

\begin{tabular}{|c|c|c|c|c|c|}
\hline & initial & D1 & D2 & D3 & D4 \\
\hline Wet weight $\mu \mathrm{g}$ & $167 \pm 6^{\mathrm{a}}$ & $298 \pm 4^{c}$ & $306 \pm 3^{c}$ & $297 \pm 10^{c}$ & $270 \pm 4^{b}$ \\
\hline Dry weight $\mu \mathrm{g}$ & $30.8 \pm 1.2^{\mathrm{a}}$ & $54.4 \pm 0.7^{\mathrm{c}}$ & $49.2 \pm 1.2^{c}$ & $45.0 \pm 1.5^{\mathrm{bc}}$ & $42.5 \pm 2.9^{b}$ \\
\hline $\begin{array}{l}\text { Survival \% } \\
(*)\end{array}$ & & $\begin{array}{c}25 \pm 19 \\
\left(35 \pm 6^{\mathrm{c}}\right)\end{array}$ & $\begin{array}{c}28 \pm 19 \\
\left(39 \pm 0.6^{c}\right)\end{array}$ & $\begin{array}{c}28 \pm 3 \\
\left(28 \pm 3^{b}\right)\end{array}$ & $\begin{array}{c}15 \pm 2 \\
\left(15 \pm 2^{a}\right)\end{array}$ \\
\hline
\end{tabular}

\footnotetext{
* survival when omitting tanks having accidental mortalities ( $\mathrm{n}=2$ for treatments $\mathrm{D} 1$ and $\mathrm{D} 2)$. Values having the
} same superscript letter do not differ in a Newman - Keuls range test $(\mathrm{P}<0.05)$

The total fatty acid content of larvae differed among treatments (Table 4), lower values were observed in larvae fed D3 and D4 and the highest value in larvae fed D1. In spite of the insignificant increase in total fatty acid content in larvae fed D3 and D4, the fatty acid profiles were deeply modified compared to initial larvae as well as for larvae fed D1 and D2, in which the total fatty acid increase was significant. Lack of n-3 HUFA in diet D4 resulted in low amount of these fatty acids in the larvae. The DHA (22:6n-3) levels were similar among larvae from treatments D1, D2 and D3, and differences in EPA (20:5n-3) corresponded to 
different dietary levels. Egg lecithin provided arachidonic acid (20:4n-6) in diets D1 and D2 and induced higher arachidonic acid content in these larvae, than in other treatments. Presence of 18:3n-6 in diets D2, D3 and D4 (supplied by Spirulina) induced higher contents of this fatty acid in larvae fed these diets, compared to treatment D1. Low levels of 20:3n-6 were observed and the highest proportion was found in larvae fed diet 4 despite the lack of this fatty acid in this diet.

Table 4: Total quantity of fatty acids (FA) per larvae and fatty acid profiles of larvae. Mean and standard error $(n=3 ; n=4$ for initial larva; $\mathrm{n}=2$ for starved larva).

\begin{tabular}{|c|c|c|c|c|c|c|}
\hline days & 3 & 8 & & 17 & & \\
\hline diets & initial & starvation & D1 & D2 & D3 & D4 \\
\hline${\text { FAt } \mu \mathrm{g} \cdot \text { larva }^{-1}}^{-1}$ & $3.8 \pm 0.3^{\mathrm{b}}$ & $2.1 \pm 0.4^{\mathrm{a}}$ & $11.5 \pm 1.2^{\mathrm{d}}$ & $6.8 \pm 0.5^{\mathrm{c}}$ & $4.8 \pm 0.3^{\mathrm{b}}$ & $4.7 \pm 0.4^{\mathrm{b}}$ \\
\hline \multicolumn{7}{|l|}{ FA ( $\%$ area $)$} \\
\hline $16: 0$ & $20.9 \pm 0.3^{\mathrm{c}}$ & $19.6 \pm 0.1^{\mathrm{bc}}$ & $20.8 \pm 1.0^{\mathrm{c}}$ & $18.7 \pm 0.5^{\mathrm{b}}$ & $17.2 \pm 0.1^{\mathrm{ab}}$ & $16.6 \pm 0.3^{\mathrm{a}}$ \\
\hline 18:0 & $6.9 \pm 0.1^{\mathrm{a}}$ & $11.0 \pm 0.5^{\mathrm{c}}$ & $8.6 \pm 0.0^{\mathrm{b}}$ & $9.0 \pm 0.6^{\mathrm{b}}$ & $8.4 \pm 0.1^{\mathrm{b}}$ & $8.5 \pm 0.0^{\mathrm{b}}$ \\
\hline $16: 1 n-7$ & $5.7 \pm 0.1^{\mathrm{c}}$ & $2.7 \pm 0.3^{\mathrm{b}}$ & $2.6 \pm 0.0^{\mathrm{b}}$ & $1.4 \pm 0.5^{\mathrm{a}}$ & $0.8 \pm 0.1^{\mathrm{ab}}$ & $0.7 \pm 0.1^{\mathrm{a}}$ \\
\hline $18: 1 n-7$ & $3.1 \pm 0.1^{\mathrm{d}}$ & $2.7 \pm 0.0^{\mathrm{c}}$ & $2.4 \pm 0.0^{\mathrm{b}}$ & $1.8 \pm 0.2^{\mathrm{a}}$ & $1.6 \pm 0.0^{\mathrm{ab}}$ & $1.8 \pm 0.0^{\mathrm{a}}$ \\
\hline $18: 1 n-9$ & $12.5 \pm 0.3^{\mathrm{b}}$ & $10.9 \pm 0.5^{\mathrm{a}}$ & $15.0 \pm 0.2^{\mathrm{c}}$ & $20.7 \pm 0.3^{\mathrm{e}}$ & $16.9 \pm 0.2^{\mathrm{d}}$ & $27.6 \pm 0.6^{\mathrm{f}}$ \\
\hline $18: 2 n-6$ & $3.3 \pm 0.1^{\mathrm{b}}$ & $2.3 \pm 0.2^{\mathrm{a}}$ & $8.0 \pm 0.1^{\mathrm{c}}$ & $7.8 \pm 0.5^{\mathrm{c}}$ & $17.3 \pm 0.2^{\mathrm{d}}$ & $25.4 \pm 0.7^{\mathrm{e}}$ \\
\hline $18: 3 n-6$ & $0.4 \pm 0.2^{\mathrm{a}}$ & $0.1 \pm 0.0^{\mathrm{a}}$ & $0.2 \pm 0.0^{\mathrm{a}}$ & $0.6 \pm 0.0^{\mathrm{b}}$ & $0.7 \pm 0.1^{\mathrm{b}}$ & $0.8 \pm 0.0^{\mathrm{b}}$ \\
\hline $20: 2 n-6$ & $0.2 \pm 0.0^{\mathrm{a}}$ & $0.2 \pm 0.0^{\mathrm{a}}$ & $0.3 \pm 0.0^{\mathrm{b}}$ & $0.4 \pm 0.0^{\mathrm{c}}$ & $0.6 \pm 0.0^{\mathrm{d}}$ & $0.6 \pm 0.0^{\mathrm{d}}$ \\
\hline $20: 3 n-6$ & $0.2 \pm 0.0^{\mathrm{ab}}$ & $0.2 \pm 0.0^{\mathrm{a}}$ & $0.2 \pm 0.0^{\mathrm{ab}}$ & $0.3 \pm 0.0^{\mathrm{b}}$ & $0.2 \pm 0.0^{\mathrm{ab}}$ & $0.3 \pm 0.0^{\mathrm{c}}$ \\
\hline $20: 4 n-6$ & $1.8 \pm 0.0^{\mathrm{b}}$ & $3.2 \pm 0.3^{c}$ & $4.1 \pm 0.2^{\mathrm{d}}$ & $4.6 \pm 0.4^{\mathrm{d}}$ & $1.3 \pm 0.0^{\mathrm{a}}$ & $1.0 \pm 0.0^{\mathrm{a}}$ \\
\hline $22: 5 n-6$ & $0.4 \pm 0.0^{\mathrm{a}}$ & $0.5 \pm 0.1^{\mathrm{a}}$ & $4.7 \pm 0.4^{\mathrm{b}}$ & $0.8 \pm 0.1^{\mathrm{a}}$ & $0.5 \pm 0.0^{\mathrm{a}}$ & $0.2 \pm 0.0^{\mathrm{a}}$ \\
\hline $18: 3 n-3$ & $0.4 \pm 0.0^{\mathrm{a}}$ & $0.3 \pm 0.1^{\mathrm{a}}$ & $0.3 \pm 0.1^{\mathrm{a}}$ & $0.3 \pm 0.1^{\mathrm{a}}$ & $1.5 \pm 0.0^{\mathrm{b}}$ & $1.7 \pm 0.0^{\mathrm{b}}$ \\
\hline $18: 4 n-3$ & $0.6 \pm 0.0^{\mathrm{b}}$ & $0.2 \pm 0.1^{\mathrm{b}}$ & $0.3 \pm 0.1^{\mathrm{b}}$ & $0.3 \pm 0.1^{\mathrm{b}}$ & $0.2 \pm 0.0^{\mathrm{b}}$ & $0.1 \pm 0.0^{\mathrm{a}}$ \\
\hline $20: 3 n-3$ & $0.2 \pm 0.0$ & $0.1 \pm 0.1$ & $0.1 \pm 0.0$ & $0.1 \pm 0.0$ & $0.2 \pm 0.0$ & $0.1 \pm 0.0$ \\
\hline $20: 4 n-3$ & $0.6 \pm 0.0^{\mathrm{c}}$ & $0.4 \pm 0.1^{\mathrm{bc}}$ & $0.3 \pm 0.1^{\mathrm{b}}$ & $0.3 \pm 0.1^{\mathrm{b}}$ & $0.2 \pm 0.0^{\mathrm{b}}$ & $0.0 \pm 0.0^{\mathrm{a}}$ \\
\hline $20: 5 n-3$ & $8.3 \pm 0.1^{\mathrm{e}}$ & $6.3 \pm 0.1^{\mathrm{d}}$ & $2.4 \pm 0.4^{\mathrm{b}}$ & $4.4 \pm 0.3^{\mathrm{c}}$ & $4.8 \pm 0.0^{\mathrm{c}}$ & $1.3 \pm 0.1^{\mathrm{a}}$ \\
\hline $22: 5 n-3$ & $2.8 \pm 0.1^{\mathrm{d}}$ & $2.1 \pm 0.1^{\mathrm{c}}$ & $0.7 \pm 0.2^{\mathrm{a}}$ & $1.4 \pm 0.2^{\mathrm{b}}$ & $1.3 \pm 0.0^{\mathrm{b}}$ & $0.6 \pm 0.0^{\mathrm{a}}$ \\
\hline $22: 6 n-3$ & $27.9 \pm 0.4^{\mathrm{c}}$ & $32.4 \pm 1.8^{\mathrm{d}}$ & $23.0 \pm 0.5^{b}$ & $23.1 \pm 1.7^{\mathrm{b}}$ & $23.8 \pm 0.6^{\mathrm{b}}$ & $11.2 \pm 0.4^{\mathrm{a}}$ \\
\hline sat & $31.4 \pm 0.5^{\mathrm{c}}$ & $32.9 \pm 0.4^{\mathrm{c}}$ & $32.3 \pm 1.1^{\mathrm{c}}$ & $29.2 \pm 0.6^{\mathrm{b}}$ & $26.9 \pm 0.1^{\mathrm{a}}$ & $26.0 \pm 0.3^{\mathrm{a}}$ \\
\hline mono & $24.2 \pm 0.5^{\mathrm{bc}}$ & $18.9 \pm 1.3^{\mathrm{a}}$ & $23.1 \pm 0.4^{\mathrm{bc}}$ & $26.4 \pm 2.2^{\mathrm{c}}$ & $20.5 \pm 0.3^{\mathrm{ab}}$ & $31.2 \pm 0.5^{\mathrm{d}}$ \\
\hline$\Sigma \mathrm{n}-6$ & $6.3 \pm 0.2^{\mathrm{a}}$ & $6.5 \pm 0.5^{\mathrm{a}}$ & $17.5 \pm 0.6^{\mathrm{c}}$ & $14.5 \pm 1.1^{\mathrm{b}}$ & $20.6 \pm 0.2^{\mathrm{d}}$ & $28.4 \pm 0.7^{\mathrm{e}}$ \\
\hline$\Sigma \mathrm{n}-3$ & $40.9 \pm 0.5^{\mathrm{d}}$ & $41.7 \pm 1.5^{\mathrm{d}}$ & $27.1 \pm 1.3^{\mathrm{b}}$ & $30.0 \pm 0.9^{c}$ & $32.1 \pm 0.6^{\mathrm{c}}$ & $15.0 \pm 0.5^{\mathrm{a}}$ \\
\hline
\end{tabular}

Values having the same superscript letter do not differ in a Newman-Keuls range test $(\mathrm{P}<0.05)$ 
The quantities of fatty acids per larvae (Table 5), allows the absolute increase or decrease of each fatty acid in final larvae compared to initial larvae to be determined. In treatment D4, 20:3n-6 and 20:2 n-6 significantly increased compared to initial content while 20:4n-6, 20:5n3 and 22:6n-3 decreased in larvae which did not received any of these fatty acids in the diet; major dietary fatty acids such as 18:1n-9 and 18:2n-6 were incorporated in high quantities into the larvae. Despite good amounts of 22:6n-3, 20:5n-3 and low amounts of 20:3n-6 and 20:4n-6 in diet D3, larvae fed on this diet did not show a significant increase in any of these fatty acids, although other major dietary fatty acids such as 18:2n-6, 18:1n9 and 18:3n-3 were clearly incorporated. Diet D3 contained also around 15\% of 16:0, and only 3\% of 18:0, while in the larvae 16:0 did not increase but 18:0 was higher than in the initial larvae. Larvae fed diets D1 and D2 exhibited significant increases in most fatty acids, including DHA, but other n-3 fatty acids including EPA did not differ from initial quantities.

The fatty acid profiles of polar lipids (Table 6) display the same dietary influences as in total lipids, with higher proportions of 22:6n-3, 20:4n-6, 16:0 and 18:0 than in total lipids, while 20:5n-3 was at similar levels in polar and total lipids for each treatment. Levels of ARA in polar lipids discriminates clearly D1 and D2 from D3 and D4 fed larvae and levels of DHA separates D1, D2 and D3 fed larvae from those fed D4. 
Table 5: Fatty acid quantities in ng.larva ${ }^{-1}$

\begin{tabular}{|c|c|c|c|c|c|c|}
\hline & day3 & day8(starved) & D1 & D2 & D3 & D4 \\
\hline $16: 0$ & $800 \pm 32 b$ & $409 \pm 52 \mathrm{a}$ & $2376 \pm 80 d$ & $1267 \pm 66 c$ & $816 \pm 3 b$ & $784 \pm 47 b$ \\
\hline 18:0 & $263 \pm 12 a$ & $230 \pm 37 a$ & $984 \pm 53 d$ & $609 \pm 30 c$ & $398 \pm 20 b$ & $400 \pm 20 b$ \\
\hline $18: 1 n-9$ & $478 \pm 23 a$ & $227 \pm 17 \mathrm{a}$ & $1715 \pm 89 \mathrm{e}$ & $1398 \pm 53 d$ & $806 \pm 37 c$ & $1304 \pm 88 d$ \\
\hline $18: 2 n-6$ & $126 \pm 3 *$ & $48 \pm 2 *$ & $916 \pm 47 b$ & $527 \pm 39 a$ & $822 \pm 41 b$ & $1201 \pm 85 c$ \\
\hline $18: 3 n-6$ & $14 \pm 6 a$ & $3 \pm 0 *$ & $23 \pm 5 \mathrm{ab}$ & $39 \pm 2 b$ & $34 \pm 1 b$ & $39 \pm 3 b$ \\
\hline $20: 2 n-6$ & $7 \pm 1 \mathrm{a}$ & $5 \pm 1 \mathrm{a}$ & $36 \pm 5 b$ & $28 \pm 3 b$ & $29 \pm 2 b$ & $29 \pm 0 \mathrm{~b}$ \\
\hline $20: 3 n-6$ & $8 \pm 1 b$ & $3 \pm 0 \mathrm{a}$ & $23 \pm 2 d$ & $17 \pm 1 \mathrm{c}$ & $10 \pm 0.0 \mathrm{~b}$ & $16 \pm 1 \mathrm{c}$ \\
\hline $20: 4 n-6$ & $70 \pm 3 b$ & $66 \pm 2 b$ & $463 \pm 24^{*}$ & $312 \pm 22 *$ & $60 \pm 3 b$ & $47 \pm 2 a$ \\
\hline $18: 3 n-3$ & $17 \pm 1 \mathrm{a}$ & $5 \pm 0 *$ & $39 \pm 11 a$ & $24 \pm 6 \mathrm{a}$ & $72 \pm 1 b$ & $80 \pm 6 b$ \\
\hline $18: 4 n-3$ & $22 \pm 1 \mathrm{ab}$ & $5 \pm 1 \mathrm{a}$ & $32 \pm 7 b$ & $24 \pm 10 \mathrm{ab}$ & $11 \pm 2 \mathrm{ab}$ & $3 \pm 2 \mathrm{a}$ \\
\hline $20: 3 n-3$ & $6 \pm 1 a b$ & $1 \pm 1 \mathrm{a}$ & $9 \pm 0 \mathrm{~b}$ & $8 \pm 1 b$ & $9 \pm 2 b$ & $5 \pm 1 \mathrm{ab}$ \\
\hline $20: 4 n-3$ & $23 \pm 2 \mathrm{ab}$ & $0 \pm 1 \mathrm{a}$ & $31 \pm 7 b$ & $21 \pm 9 \mathrm{ab}$ & $11 \pm 1 \mathrm{ab}$ & $1 \pm 1 \mathrm{a}$ \\
\hline $20: 5 n-3$ & $318 \pm 16 b$ & $131 \pm 15 \mathrm{a}$ & $280 \pm 59 b$ & $300 \pm 31 b$ & $229 \pm 8 b$ & $62 \pm 6 a$ \\
\hline $22: 5 n-3$ & $107 \pm 4 c$ & $43 \pm 3 a b$ & $82 \pm 23^{*}$ & $96 \pm 14^{*}$ & $64 \pm 3 a b c$ & $28 \pm 0 \mathrm{a}$ \\
\hline $22: 6 n-3$ & $1067 \pm 49 b$ & $681 \pm 120 \mathrm{a}$ & $2644 \pm 204 d$ & $1552 \pm 69 \mathrm{c}$ & $1131 \pm 64 b$ & $526 \pm 18 \mathrm{a}$ \\
\hline $\mathrm{n}-3 \mathrm{int} * *$ & $471 \pm 22 \mathrm{c}$ & $187 \pm 16 a b$ & $424 \pm 96 c$ & $441 \pm 64 c$ & $317 \pm 13 b c$ & $93 \pm 8 \mathrm{a}$ \\
\hline
\end{tabular}

Values having the same superscript letter do not differ in a Newman-Keuls range test $(\mathrm{P}<0.05)$

* treatments omitted having unequal variances, the ANOVA was performed on remaining treatments. ** sum of n-3 intermediates of elongation-desaturation (18:4, 20:4, 20:5, 22:5 n-3) 
Table 6 : Fatty acid profile of polar lipids in larvae ( $\%$ area $)$.

\begin{tabular}{|c|c|c|c|c|c|}
\hline & initial & D1 & $\mathrm{D} 2$ & D3 & D4 \\
\hline $16: 0$ & $23.3 \pm 0.3^{\mathrm{d}}$ & $23.1 \pm 0.6^{d}$ & $21.6 \pm 0.4^{\mathrm{c}}$ & $19.5 \pm 0.3^{b}$ & $18.2 \pm 0.5^{\mathrm{a}}$ \\
\hline 18:0 & $8.7 \pm 0.3^{\mathrm{a}}$ & $10.5 \pm 0.4^{b}$ & $11.0 \pm 0.2^{b}$ & $10.4 \pm 0.1^{\mathrm{b}}$ & $10.2 \pm 0.1^{b}$ \\
\hline $16: 1 n-7$ & $2.3 \pm 0.1^{\mathrm{c}}$ & $1.0 \pm 0.0^{b}$ & $0.8 \pm 0.1^{\mathrm{ab}}$ & $0.5 \pm 0.0^{\mathrm{a}}$ & $0.8 \pm 0.2^{\mathrm{ab}}$ \\
\hline $18: 1 n-7$ & $2.6 \pm 0.1^{\mathrm{c}}$ & $2.0 \pm 0.1^{b}$ & $1.8 \pm 0.1^{\mathrm{a}}$ & $1.7 \pm 0.1^{\mathrm{a}}$ & $2.2 \pm 0.1^{\mathrm{b}}$ \\
\hline $18: 1 n-9$ & $9.0 \pm 0.1^{\mathrm{a}}$ & $10.5 \pm 0.3^{b}$ & $14.8 \pm 0.4^{\mathrm{d}}$ & $12.7 \pm 0.1^{\mathrm{c}}$ & $19.0 \pm 0.3^{\mathrm{e}}$ \\
\hline $18: 2 n-6$ & $2.2 \pm 0.1^{\mathrm{a}}$ & $6.4 \pm 0.2^{b}$ & $6.9 \pm 0.1^{b}$ & $13.5 \pm 0.4^{\mathrm{c}}$ & $24.7 \pm 0.2^{d}$ \\
\hline $18: 3 n-6$ & $0.1 \pm 0.0^{\mathrm{a}}$ & $0.1 \pm 0.0^{\mathrm{a}}$ & $0.3 \pm 0.0^{\mathrm{ab}}$ & $0.5 \pm 0.2^{b c}$ & $0.8 \pm 0.2^{c}$ \\
\hline $20: 2 n-6$ & $0.3 \pm 0.0^{\mathrm{a}}$ & $0.3 \pm 0.1^{\mathrm{a}}$ & $0.4 \pm 0.0^{\mathrm{a}}$ & $0.7 \pm 0.0^{b}$ & $0.9 \pm 0.1^{b}$ \\
\hline $20: 3 n-6$ & $0.1 \pm 0.0^{\mathrm{a}}$ & $0.2 \pm 0.0^{\mathrm{b}}$ & $0.3 \pm 0.0^{\mathrm{c}}$ & $0.2 \pm 0.0^{\mathrm{c}}$ & $0.4 \pm 0.0^{\mathrm{d}}$ \\
\hline $20: 4 n-6$ & $2.2 \pm 0.0^{\mathrm{c}}$ & $5.9 \pm 0.0^{\mathrm{d}}$ & $5.9 \pm 0.0^{\mathrm{d}}$ & $1.6 \pm 0.0^{b}$ & $1.3 \pm 0.1^{\mathrm{a}}$ \\
\hline $22: 5 n-6$ & $0.4 \pm 0.0^{b}$ & $5.9 \pm 0.2^{\mathrm{e}}$ & $1.0 \pm 0.1^{\mathrm{d}}$ & $0.6 \pm 0.0^{\mathrm{c}}$ & $0.2 \pm 0.0^{\mathrm{a}}$ \\
\hline $18: 3 n-3$ & $0.2 \pm 0.0^{\mathrm{a}}$ & $0.1 \pm 0.0^{\mathrm{a}}$ & $0.1 \pm 0.0^{\mathrm{a}}$ & $0.7 \pm 0.1^{b}$ & $1.4 \pm 0.2^{\mathrm{c}}$ \\
\hline $18: 4 n-3$ & $0.2 \pm 0.0$ & $0.1 \pm 0.0$ & $0.1 \pm 0.0$ & $0.2 \pm 0.1$ & $0.2 \pm 0.1$ \\
\hline $20: 3 n-3$ & $0.05 \pm 0.0^{b}$ & $\mathrm{nd} \pm 0.0^{\mathrm{a}}$ & $0.1 \pm 0.0^{b}$ & $0.1 \pm 0.0^{\mathrm{b}}$ & $0.1 \pm 0.0^{\mathrm{b}}$ \\
\hline $20: 4 n-3$ & $0.3 \pm 0.0^{\mathrm{d}}$ & $0.1 \pm 0.0^{\mathrm{b}}$ & $0.1 \pm 0.0^{b}$ & $0.2 \pm 0.0^{\mathrm{c}}$ & $0.05 \pm 0.0^{\mathrm{a}}$ \\
\hline $20: 5 n-3$ & $8.3 \pm 0.1^{\mathrm{e}}$ & $2.2 \pm 0.0^{b}$ & $4.2 \pm 0.1^{\mathrm{c}}$ & $5.0 \pm 0.1^{\mathrm{d}}$ & $1.6 \pm 0.1^{\mathrm{a}}$ \\
\hline $22: 5 n-3$ & $2.4 \pm 0.1^{\mathrm{e}}$ & $0.5 \pm 0.0^{\mathrm{a}}$ & $1.1 \pm 0.0^{\mathrm{c}}$ & $1.3 \pm 0.0^{\mathrm{d}}$ & $0.7 \pm 0.0^{\mathrm{b}}$ \\
\hline $22: 6 n-3$ & $34.6 \pm 0.5^{c}$ & $28.7 \pm 1.0^{b}$ & $28.3 \pm 0.9^{b}$ & $29.2 \pm 1.0^{b}$ & $15.2 \pm 0.3^{\mathrm{a}}$ \\
\hline sat & $33.6 \pm 0.5^{b}$ & $34.6 \pm 0.9^{b}$ & $32.9 \pm 0.4^{b}$ & $30.4 \pm 0.2^{\mathrm{a}}$ & $29.0 \pm 0.4^{\mathrm{a}}$ \\
\hline mono & $15.1 \pm 0.2^{\mathrm{a}}$ & $14.7 \pm 0.2^{\mathrm{a}}$ & $18.3 \pm 0.6^{b}$ & $15.8 \pm 0.3^{\mathrm{a}}$ & $23.3 \pm 0.4^{\mathrm{c}}$ \\
\hline$\Sigma \mathrm{n}-6$ & $5.3 \pm 0.1^{\mathrm{a}}$ & $18.9 \pm 0.1^{\mathrm{d}}$ & $14.7 \pm 0.0^{b}$ & $17.1 \pm 0.5^{\mathrm{c}}$ & $28.4 \pm 0.1^{\mathrm{e}}$ \\
\hline$\sum \mathrm{n}-3$ & $46.0 \pm 0.5^{\mathrm{e}}$ & $31.8 \pm 1.0^{b}$ & $34.1 \pm 1.0^{\mathrm{c}}$ & $36.7 \pm 0.9^{\mathrm{d}}$ & $19.2 \pm 0.2^{\mathrm{a}}$ \\
\hline
\end{tabular}

Values having the same superscript letter do not differ in a Newman-Keuls range test $(\mathrm{P}<0.05)$

\section{Discussion}

Final dry weights obtained during this experiment were lower than gilthead seabream larvae fed rotifers (Fernandez-Diaz and Yufera, 1997; Yufera et al., 1999), which can reach around $80 \mu \mathrm{g}$ dry weight at day 15 . However, effective growth and survivals were obtained with the various diets even with larvae fed the HUFA depleted diet D4. The general husbandry technology has to be improved, but can be considered as sufficient for experimental purposes. 
The diet D1 used as control in the present study gave results in larval rearing similar to a corresponding diet tested in a previous study (Robin and Vincent 2003). However, this diet is not very useful as a semi-purified diet to study lipid requirements since one ingredient (Algamac, a Schizochytrium powder) is rich in DHA. Replacement of Algamac by Spirulina is a way to have a basal diet without highly unsaturated fatty acids and therefore more convenient for nutrition studies. Comparison of results obtained with diet D1 and D2 let to observe that this replacement had not no significant effect on larval growth or survival. As also observed by Robin and Vincent (2003), diet D1 containing Algamac induced high lipid content into larvae (measured here as total fatty acid) compared to other treatments. The effects of dietary long chain essential fatty acids can be observed by comparison of treatments D2, D3 and D4. As with most marine fish, gilthead seabream is considered to have an absolute requirement for n-3 highly unsaturated fatty acids like EPA and DHA (Rodriguez et al., 1998). A reduction of growth and survival normally result from feeding marine fish larvae on a diet with low HUFA content. Such influences were observed here with the HUFA depleted diet D4. As demonstrated by Bessonart et al. (1999), arachidonic acid is also required by this species. In the present experiment, the influence of low arachidonic content of diet D3, compared to diet D2, did not lead to a significant reduction in larval growth, however survival obtained with D3 was lower than with D2 (when accidental mortalities were not included). Low dietary arachidonic acid was supplied by diet 3, but the quantity of this fatty acid did not increase in the larvae, in fact there was a tendency to decrease $(\mathrm{P}<0.08)$ compared to initial quantity, despite a theoretical preferential incorporation of this fatty acid (Linares and Henderson, 1991). Larvae fed on diet D3 can thus be considered as deficient in arachidonic acid. The lower total fatty acid contents, reflecting lower lipid stores, of larvae fed diet D3 (and diet D4) compared to D2, can also be considered as a sign of a lower nutritional status related to dietary arachidonic acid deficiency. As generally occurs (Linares 
and Henderson, 1991), DHA and arachidonic acid were incorporated preferentially into polar lipids. EPA remained at similar proportion in polar lipids than in total lipids, with differences between treatments corresponding to diet content.

Although the growth of larvae fed diets D3 and D4 was indeed occurring, there was no significant increase in total fatty acids per larvae compared to the initial group, just before feeding. However the fatty acid profiles of these larvae were highly affected by dietary fatty acid profiles, as well as for larvae fed diets D1 and D2. Differences in total lipid content between larvae did not seem to affect the response in their fatty acid profile to dietary fatty acid pattern. For instance, with diets D1, D2 and D3 providing the same DHA level, the results in larval DHA level in total lipids as well as in polar lipids did not differ between these treatments, while corresponding values were 50\% lower in larvae fed diet D4. As a result of dietary fatty acid influences, despite insignificant total fatty acid (FA) increase in larvae fed diet D3 and D4 versus initial larvae, significant changes occurred in quantities of individual fatty acid per larvae. In case of treatment D3, the mean increase of 18:2n-6 plus 18:1n-9 plus 18:3n-3 represented 1.1 $\mu \mathrm{g}$. larvae $^{-1}$ while the total FA increase was $0.9 \mu \mathrm{g}$. larvae $^{-1}$. For treatment D4, the mean increase of the same FAs represented 2.1 $\mu \mathrm{g}$.larvae ${ }^{-1}$ while the total FA increase was only $0.9 \mu \mathrm{g} \cdot$ larvae $^{-1}$ due to the decrease of other fatty acids, mainly n-3 HUFA. This suggest that a turn-over of fatty acid occured in larvae during the experiment, with consumption of endogenous lipids being replaced by incorporation of dietary fatty acids. The use of Spirulina in the basal diet supplied some 18:3n-6 : the $\Delta 6$ desaturation product of 18:2n-6. The presence of $18: 3 n-6$ is useful to check if elongation into $20: 3 n-6$ and $\Delta 5$ desaturation into 20:4n-6 occurs. The requirement for highly unsaturated fatty acid of marine fish species was interpreted as an inability to produce these fatty acids from the respective precursors, 18:3n-3 and 18:2n-6. This question is now revised. By injecting labelled n-3 precursors, Mourente and Tocher (1994) have demonstrated there was $\Delta 6$ and $\Delta 5$-desaturase 
activities in starved gilthead seabream juvenile. Seiliez et al. (2003) identified a $\Delta 6$-desaturase in gilthead seabream fed on a HUFA-free diet, the fatty acid profile of so fed fish was characterized by high levels of 18:2n-9 ( $\Delta 6$-desaturation product of dietary $18: 1 n-9)$ and some $20: 2 n-9$ the elongation product of the former, these products as well as desaturase mRNA were not observed in control fish fed with a diet containing n-3 HUFA. As it was also shown in salmonids (Ruyter and Tomassen, 1999; Seiliez et al. 2001), dietary HUFA may repress desaturase expression. In the present study larvae fed diet D4 did not show any accumulation of n-3 fatty acids other than 18:3n-3, and the quantities of EPA and DHA decreased in comparison with initial larvae, then no desaturation products appeared. In the n- 6 series the diet D4 provided 18:3n-6 which increased in fish as well as its elongation product 20:3n-6, however the total quantity of arachidonic acid (20:4n-6) decreased compared to initial larvae, indicating that elongation of $18: 3 n-6$ to $20: 3 n-6$ occurs, but no sign of $\Delta 5$-desaturation is observed. Tocher and Ghioni (1999) already mentioned a low $\Delta 5$-desaturation capacity in this species. 20:2n-6, the product of elongation of 18:2n-6 also increased, but this fatty acid cannot be used for further transformation. Comparison of 20:3n- 6 quantities in larvae fed D3 and D4 may also suggest an inhibition of elongation from 18:3n-6 when larvae were fed D3 with high level of n-3 HUFA. However with such methodology, we cannot reject the hypothesis that some desaturation occurred at low rates but was masked by a higher loss of long chain fatty acids than the desaturation-elongation pathway might produce. This finding may be supported by the observation of the sum of $n-3$ intermediate fatty acids (18:4, 20:4, 20:5, 22:5), endogenous in larvae fed diet 4 , which decreased at higher rate than 22:6n-3 and could have been used to produce 22:6n-3, partly compensating its consumption. In the other treatments, the same intermediate fatty acids did not increase despite dietary inputs, mainly in EPA (20:5n-3), which might be used to form 22:6n-3. Mourente and Tocher (1994) evidenced transformation of labelled 20:5n-3 to 22:6n-3 in juvenile gilthead seabream. 
This consumption of long chain unsaturated fatty acid is a major conclusion of this study.

From the evolution of fatty acids of larvae fed diet D4, we may estimate a rate of loss around 1.1, 0.5 and $0.05 \mathrm{mg} \cdot \mathrm{day}^{-1} \cdot \mathrm{g}^{-1}$ mean dry body weight of larvae for DHA, EPA and arachidonic acid, respectively. These losses seem relatively high but may even be underestimated in the hypothesis of a partial compensation by a low desaturation capacity. In contrast, these losses might be enhanced by physiological perturbation in fish under nutritional deficiency. Koven et al. (1989) observed a decrease of DHA in dry weight basis in larvae fed Isochrysis-enriched rotifers, however this decrease was relative to growth and dry weight increase. In the present study, the methodology used allows us to observe that it corresponded to a net loss. Losses of these fatty acids were already observed in larvae during fasting (Koven et al., 1989; Tandler et al., 1989: Mourente and Odriozola, 1990; Rønnestad et al., 1994) but in such case these decreases could be attributed to energy demand. In the present study, larvae fed D4 did not suffer from an energy deficiency since they were able to accumulate non essential fatty acids $(18: 0,18: 1 n-9)$ or PUFA (18:2n-6, 18:3n-3). Starved larvae in the present experiment had a relative decrease in DHA and EPA per day about twice as higher than in larvae fed HUFA depleted diet D4. The low increase of n-3 HUFA compared to other major dietary fatty acids, observed in larvae fed on the diet D3, could be explained by such consumption of endogenous n-3 HUFA occurring at the same time than incorporation of dietary n-3 HUFA. Such turnover may explain also the low relative incorporation of $n-3$ fatty acids mentioned by Robin and Vincent (2003) in larvae of this species. 


\section{Acknowledgments}

This work was supported as a part of EU project Q5RS-2000-30058 (RAFOA). The authors are grateful to J.-S. Bruant (FMD, La Brée les Bains, France) who provided spawn as a favour, to P. Quazuguel and H. Le Delliou for their assistance, to P. Bergot and S. Kaushik (INRA, St Pee sur Nivelle, France) for sound advice and English corrections.

\section{References}

Antueno, R.J. de, Knickle, L. C., Smith, H., Elliot, M.L., Allen, S.J., Nwaka, S., Winther, M.D., 2001. Activity of human $\triangle 5$ and $\Delta 6$ desaturases on multiple n-3 and n-6 polyunsaturated fatty acids. FEBS Letters 509, 77-80

Bessonart, M., Izquierdo, M. S., Salhi, M., Hernández-Cruz, C. M., González, M. M., Fernández-Palacios, H., 1999. Effect of dietary arachidonic acid levels on growth and survival of gilthead sea bream (Sparus aurata L.) larvae. Aquaculture 179, 265-275.

Cahu, C.L., Zambonino-Infante, J.L., 2001. Substitution of live food by formulated diets in marine fish larvae. Aquaculture 200, 161-180.

Cahu, C.L., Zambonino Infante, J.L., Barbosa, V., 2003. Effect of dietary phospholipid level and phospholipid:neutral lipid value on the development of sea bass (Dicentrarchus labrax) larvae fed a compound diet. Br. J. Nutr. 90, 21-28

Carvalho, A.P., Escaffre, A.-M., Oliva Teles, A., Bergot, P., 1997. First feeding of common carp larvae on diets with high levels of protein hydrolysates. Aquacult. Int. 5, 361-367.

Fernández-Díaz, C., Yúfera, M., 1997. Detecting growth in gilthead seabream, Sparus aurata L., larvae fed microcapsules. Aquaculture 153, 93-102

Folch, J., Lees, M., Sloane-Stanley, G.H., 1957. A simple method for the isolation and purification of total lipids from animal tissues. J. Biol. Chem. 226, 497-509. 
Fontagné, S., Robin, J., Corraze, G., Bergot, P., 2000. Growth and survival of European sea bass (Dicentrarchus labrax) larvae fed from first feeding on compound diets containing medium-chain triacylglycerols. Aquaculture 190, 261-271

Ghioni, C , Tocher, D.R., Bell, M.V., Dick, J.M., Sargent, J.R., 1999. Low C18 to C20 fatty acid elongase activity and limited conversion of stearidonic acid, 18:4(n-3), to eicosapentaenoic acid, 20:5(n-3), in a cell line from turbot, Scophthalmus maximus. Biochim. Biophys. Acta 1437, 170-181.

Juaneda, P., Rocquelin, G., 1985. Rapid and convenient separation of phospholipids and nonphosphorus lipids from rat heart using silica cartridges. Lipids 20, 40-41.

Kanazawa, A., 1985. Essential fatty acid and lipid requirement of fish. In: Cowey, C.B., Mackie, A.M., Bell, J.G. (Eds.) Nutrition and Feeding in Fish. Academic Press, London, pp. 281-298.

Koven, W.M., Kissil, G. W., Tandler, A., 1989. Lipid and n-3 requirement of Sparus aurata larvae during starvation and feeding. Aquaculture, 79, 185-191.

Koven, W., Kolkovski, S., Hadas, E., Gamsiz, K., Tandler A., 2001. Advances in the development of microdiets for gilthead seabream, Sparus aurata: a review. Aquaculture $194,107-121$.

Leonard, A. E., Pereira, S. L., Sprecher, H., Huang, Y-S., 2004. Elongation of long-chain fatty acids. Prog. Lipid Res. 43, 36-54

Linares, F., Henderson, R.J., 1991. Incorporation of 14C-labelled polyunsaturated fatty acids by juvenile turbot, Scophthalmus maximus (L.) in vivo. J. Fish Biol. 38, 335-347.

Morrisson, W., Smith, L., 1964. Preparation of fatty acid methyl esters and dimethylacetals from lipids with boron fluoride-methanol. J. Lipid Res. 3, 600-608 
Mourente, G., Odriozola, J.M., 1990. Effect of broodstock diets on total lipid and fatty acid composition of gilthead sea bream (Sparus aurata L.) during yolk sac stage. Fish Physiol. Biochem. 8, 103-110.

Mourente, G., Tocher, D.R., 1994. In vivo metabolism of [1-14C] linolenic acid (18:3(n-3)) and [1-14C] eicosapentaenoic acid $(20: 5(n-3))$ in marine fish: Time-course of the desaturation/elongation pathway. Biochim. Biophys. Acta 1212, 109-118.

Radünz-Neto, J., Corraze, G., Charlon, N., Bergot, P., 1994. Lipid supplementation of caseinbased purified diets for carp (Cyprinus carpio L.) larvae. Aquaculture 128, 153-161.

Radünz Neto, J., Corraze, G., Charlon, N., Bergot, P., Kaushik, S.J., 1996. Estimation of essential fatty acid requirements of common carp larvae using semi-purified artificial diets. Arch. Anim. Nutr. 49, 41-48

Regost, C., Arzel, J., Robin, J., Rosenlund, G., Kaushik, S.J., 2003. Total replacement of fish oil by soybean oil or linseed oil with return to fish oil in turbot (Psetta maxima). 1 growth performance, flesh fatty acid profile and lipid metabolism. Aquaculture 217, 191-199.

Robin, J. H., Vincent, B., 2003. Microparticulate diets as first food for gilthead sea bream larva (Sparus aurata): study of fatty acid incorporation. Aquaculture 225, 463-474

Rodriguez, C., Perez, J.A., Badia, P., Izquierdo, M.S., Fernandez-Palacios, H., Hernandez, A.L., 1998. The n-3 highly unsaturated fatty acids requirements of gilthead sea bream (Sparus aurata L.) larvae when using an appropriate DHA/EPA ratio in the diet. Aquaculture 169, 9-23.

Rønnestad, I., Koven, W.M., Tandler, A., Harel, M., Fyhn, H.J., 1994. Energy metabolism during development of eggs and larvae of gilthead seabream (Sparus aurata). Mar. Biol. $120,187-196$. 
Ruyter, B., Thomassen, S., 1999. Metabolism of n-3 and n-6 fatty acids in Atlantic salmon liver: stimulation by essential fatty acid deficiency. Lipids 34, 1167-1176.

Sargent, J.R., Bell, J.G., Bell, M.V., Hendersen, R.J., Tocher, D.R., 1995. Requirement criteria for essential fatty acids. J. Appl. Ichthyol. 11, 1983-198.

Seiliez, I., Panserat, S., Kaushik, S., Bergot, P., 2001. Cloning, tissue distribution and nutritional regulation of a delta 6-desaturase-like enzyme in rainbow trout. Comp. Biochem. Physiol. 130 B, 83-93

Seiliez, I., Panserat, S., Corraze, G., Kaushik, S., Bergot P., 2003. Cloning and nutritional regulation of a $\Delta 6$-desaturase-like enzyme in the marine teleost gilthead seabream (Sparus aurata). Comp. Biochem. Physiol 135B, 449-460.

Tandler, A., Watanabe, T., Satoh, S., Fukusho, K, 1989. The effect of food deprivation on the fatty acid and lipid profile of red sea bream larvae (Pagrus major). Br. J. Nutr. 62, 349361

Tocher, D.R., Ghioni, C., 1999. Fatty acid metabolism in marine fish: low activity of fatty acyl delta 5 desaturation in gilthead sea bream (Sparus aurata) cells. Lipids 34, 433440.

Yúfera, M., Pascual, E., Ferdinand-Diaz, C., 1999. A high efficient microencapsulated food for rearing early larvae of marine fish. Aquaculture 177, 249-256

Yúfera, M., Fernandez-Diaz, C., Pascual, E., Sarasquete, M., Moyano, F., Diaz, M., Alarcon, F., Garcia-Gallego, M., Parra, G., 2000. Towards an inert diet for first-feeding gilthead sea bream Sparus aurata larvae. Aquacult. Nutr. 6, 143-152. 